\title{
Hirntod und Umgang mit (potenziellen) Organspendern und Angehörigen
}

Matthias N. Ungerer, Silvia Schönenberger, Ana Paula Barreiros, Anne-Bärbel Blaes-Eise, Axel Rahmel

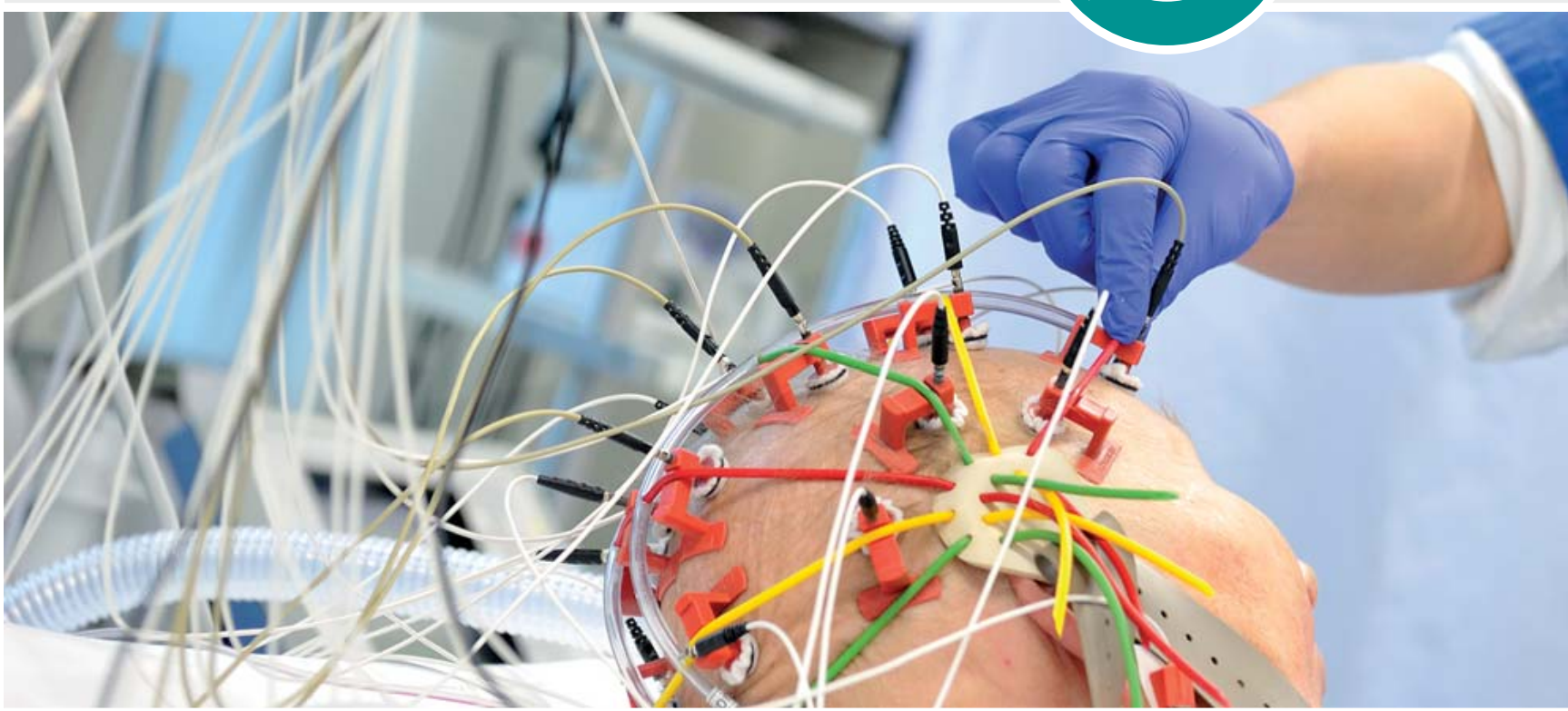

Quelle: KH Krauskopf.

In den letzten Jahren hat die Diagnose des irreversiblen Hirnfunktionsausfalls bei schwer hirngeschädigten Patienten an Bedeutung gewonnen. Der Hirntod, definiert als nicht behebbarer Ausfall der Gesamtfunktion des Großhirns, des Kleinhirns und des Hirnstamms, ist Voraussetzung zur Organentnahme im Rahmen einer Organspende. Der Artikel stellt die gesetzlichen und organisatorischen Rahmenbedingungen vor.

\section{Einleitung}

Die Diagnose des irreversiblen Hirnfunktionsausfalls ist nicht nur in der neurologischen Intensivmedizin ein wichtiger und unverzichtbarer Teilaspekt in der Behandlung schwer hirngeschädigter Patienten. Die Diagnose des irreversiblen Hirnfunktionsausfalls des Gesamthirns ist zwar ein essenzieller Bestandteil der Organspende und damit auch der Transplantationsmedizin. Darüber hinaus hat sie jedoch auch in der Behandlung und Diagnostik und v.a. im Rahmen einer Therapiezieländerung und Fortführung der intensivmedizinischen Behandlung zunehmend an Bedeutung gewonnen. So wird ca. nur jede zweite Diagnostik des irreversiblen Hirnfunktionsausfalls im Rahmen einer möglichen Organspende durchgeführt.

$\begin{array}{ll}\text { ABKÜRZUNGEN } \\ \text { ADH } & \text { antidiuretisches Hormon } \\ \mathrm{BGA} & \text { Blutgasanalyse } \\ \mathrm{CTA} & \text { CT-Angiografie } \\ \mathrm{DSO} & \text { Deutsche Stiftung Organtransplantation } \\ \mathrm{EEG} & \text { Elektroenzephalogramm } \\ \mathrm{EfA} & \text { Entscheidungsbegleitung für Angehörige } \\ \mathrm{EVD} & \text { extraventrikuläre Drainage } \\ \mathrm{ICP} & \text { intrakranieller Druck } \\ \mathrm{IHA} & \text { irreversibler Hirnfunktionsausfall } \\ \mathrm{MAP} & \text { mittlerer arterieller Blutdruck } \\ \mathrm{PaCO}_{2} & \text { arterieller Kohlendioxidpartialdruck } \\ \mathrm{PaO}_{2} & \text { arterieller Sauerstoffpartialdruck } \\ \mathrm{S}_{\mathrm{p}} \mathrm{O}_{2} & \text { pulsoxymetrisch gemessene Sauerstoffsättigung } \\ \mathrm{TPG} & \text { Transplantationsgesetz }\end{array}$




\section{FALLBEISPIEL}

Eine 42-jährige Patientin wird aufgrund eines plötzlichen Kopfschmerzereignisses vom Notarzt intubiert auf eine neurologische Intensivstation aufgenommen. In der CT inklusive CTA bei Aufnahme zeigt sich eine ausgedehnte Subarachnoidalblutung aus einem linksseitigen Mediaaneurysma mit deutlichem Liquoraufstau.

Es erfolgt zunächst die Anlage einer extraventrikulären Drainage (EVD). Im Laufe der anschließend durchgeführten diagnostischen Angiografie kommt es zu einem plötzlichen Anstieg des intrakraniellen Druckes (ICP) auf Werte $>40 \mathrm{mmHg}$ mit einem erhöhten Förderbedarf der EVD. Die Patientin erhält bei weiten, lichtstarren Pupillen und Verdacht auf eine untere Einklemmung eine hirndrucksenkende Therapie mit Osmofundin, hypertonem $\mathrm{NaCl}$ und Thiopental.

Die Patientin wird anschließend in den OP-Saal überführt, wo eine notfallmäßige operative Versorgung (Clipping) des Aneurysmas erfolgen soll. Der operative Eingriff verläuft frustran, sodass die Patientin mit unversorgtem Aneurysma und dauerhaftem ICP $>40 \mathrm{mmHg}$ auf die Station zurückverlegt wird.

Die infauste Prognose bei weiterhin erhöhtem ICP und nicht zu versorgender aneurysmatischer Blutung wird mit den Angehörigen besprochen.

\section{RECHTLICHE RAHMENBEDINGUNGEN}

Neues im 2. Gesetz zur Änderung des Transplantationsgesetzes von 2019 [1]:

- Freistellung der Transplantationsbeauftragten auf Grundlage der Anzahl der Intensivbetten

- bessere Vergütung der Entnahmekrankenhäuser

- Einrichtung eines bundesweiten neurologischen/ neurochirurgischen konsiliarärztlichen Rufbereitschaftsdienstes

- Etablierung interner Qualitätssicherungssysteme zur Erfassung einer nicht erfolgten Feststellung oder Meldung eines irreversiblen Hirnfunktionsausfalls

\section{Definition des irreversiblen Hirnfunktionsausfalls}

Der irreversible Hirnfunktionsausfall wird in der aktuellsten Fortschreibung der Richtlinie des Transplantationsgesetzes von 2015 von der Bundesärztekammer definiert als „nicht behebbarer Ausfall der Gesamtfunktion des Großhirns, des Kleinhirns und des Hirnstamms“ [2]. Die aufgeführten Richtlinien zu den Voraussetzungen und zur Durchführung der Diagnose des irreversiblen Hirnfunktionsausfalls beziehen sich auf die Veröffentlichung von Brandt et al. [3]. Unterschieden wird zwischen

- der primären Hirnschädigung durch z. B. Ischämien, Blutungen, Hirntumoren oder Traumafolgen und

- der sekundären Hirnschädigung, die als indirekte Einwirkung durch Intoxikationen, Hypoxie oder HerzKreislauf-Versagen entsteht.

Der Nachweis des Hirnfunktionsausfalls erfolgt in drei Schritten (s. a. Infobox): Zunächst müssen die Voraussetzungen für den Beginn der Diagnostik erfüllt sein, dann erfolgt die Durchführung der klinischen Untersuchung und ggf. der apparativen Untersuchungen und abschließend der Nachweis der Irreversibilität der Hirnschädigung.

\section{PRAXIS}

\section{Drei Schritte zur Diagnose des Funktionsausfalls}

- Prüfung der Voraussetzungen vor Beginn der Diagnostik

- Durchführung der klinischen und ggf. der apparativen Untersuchungen

- Notwendigkeit des Irreversibilitätsnachweises

\section{RECHTLICHE RAHMENBEDINGUNGEN}

Neues im Transplantationsgesetz von 2015

- neue Definition des Hirntodes als irreversibler, vollständiger Hirnfunktionsausfall

- neue ärztliche Qualifikationsanforderungen

- Etablierung der Duplexsonografie und CT-Angiografie zum Nachweis des zerebralen Zirkulationsstillstands 


\section{Diagnose des irreversiblen Hirnfunktionsausfalls}

\section{Voraussetzungen}

\section{PRAXIS}

\section{Voraussetzungen für die Diagnostik des Hirntodes}

- Nachweis einer Hirnschädigung:

- primär/sekundär

- supra- oder infratentoriell

- Ausschluss einer Intoxikation (Anamnese, ggf. toxikologisches Screening)

- Ausschluss eines medikamentös induzierten Komas

- Propofol und Remifentanil: > $8 \mathrm{~h}$ absetzen

- Dormicum und Sufentanil: > $12 \mathrm{~h}$ absetzen

- ggf. Spiegelbestimmung, z. B. bei Thiopental

- Cave: Reversible Ursachen für einen komatösen Zustand sind zwingend vor der Hirntoddiagnostik auszuschließen.

- Ausschluss eines metabolisch oder endokrin induzierten Komas (z. B. Elektrolyte, ggf. Ammoniak, bestimmen)

- Ausschluss eines Kreislaufschocks (z. B. bei Sepsis)

- Ausschluss primäre/therapeutische Hypothermie:

- Körpertemperatur $>35^{\circ} \mathrm{C}$ bei Einfluss zentral wirksamer Substanzen

- Cave: Nach therapeutischer Hypothermie ist frühestens $>24$ h nach Erreichen der Normothermie die Hirntoddiagnostik möglich!

Voraussetzungen für die Durchführung einer Hirntoddiagnostik sind

- das Vorliegen eines vollständigen Bewusstseinsverlusts (Koma),

- Hirnstammareflexie und

- Atemstillstand (Apnoe) des Patienten.

Jedoch müssen zuvor zwingend reversible Ursachen für einen komatösen Zustand des Patienten, wie z. B. Intoxikationen oder die Wirkung bestimmter Sedativa, vor Beginn der Diagnostik ausgeschlossen werden. Gegebenenfalls sollte die Bestimmung der Blutspiegel, z. B. nach Thiopental-Gabe, vor Beginn der Diagnostik erfolgen.

Sobald die Voraussetzungen für den Beginn der Diagnostik erfüllt sind, sollte - nach Ansicht der Autoren - im Idealfall die Deutsche Stiftung Organtransplantation (DSO) frühzeitig informiert werden, um die behandelnden Ärzte bereits beim Gespräch mit den Angehörigen zur Feststellung des mutmaßlichen Patientenwillens und dann im weiteren Ablauf möglichst effektiv unterstützen zu können.
TIPP

Entwickelt ein Patient aufgrund der Schwere der Hirnschädigung mit hoher Wahrscheinlichkeit einen irreversiblen Hirnfunktionsausfall und kommt somit ggf. für eine Organspende in Frage, so sollten bereits frühzeitig die interne Rücksprache im Team und ein ausführliches Gespräch mit den Angehörigen des Patienten über den mutmaßlichen Patientenwillen vor Einleitung der weiteren Diagnostik erfolgen.

Die Prüfung und Feststellung des irreversiblen Hirnfunktionsausfalls ist aber auch in Fällen, in denen eine Organtransplantation nicht möglich ist oder nicht dem mutmaßlichen Patientenwillen entspricht, von großer Bedeutung und kann für die Entscheidung zur Therapiebeendigung maßgeblich sein.

\section{Personelle Voraussetzungen zur Feststellung des irreversiblen Hirnfunktionsausfalls}

Der irreversible Hirnfunktionsausfall muss von zwei unabhängigen approbierten Fachärzten mit einer mehrjährigen Erfahrung in der Behandlung von schwer hirngeschädigten Patienten festgestellt werden. Zusätzlich muss einer der beiden Fachärzte entweder Facharzt für Neurologie oder Neurochirurgie sein. Als Besonderheit muss bei Patienten vor dem 14. Lebensjahr einer der beiden Untersucher Facharzt für Kinder- und Jugendmedizin sein.

\section{Klinische Untersuchung}

\section{Drei-Stufen-Schema}

Die Feststellung erfolgt auf Basis der aktuellsten Fortschreibung der Richtlinie der Bundesärztekammer anhand eines Drei-Stufen-Schemas.

- Als Erstes ist für die Diagnose des irreversiblen Hirnfunktionsausfalls der Nachweis einer akuten, schwerwiegenden Hirnschädigung unter Ausschluss möglicher reversibler Ursachen notwendig.

- Anschließend müssen die klinischen Zeichen für den Hirnfunktionsausfall erfüllt werden (s.u.). Der ApnoeTest ist dabei ein spezieller klinischer Test zum Nachweis des Ausfalls der Spontanatmung (s. Infobox).

- Als drittes Kriterium muss die Irreversibilität des Funktionsausfalls bei Erwachsenen und Kindern ab dem 2. Lebensjahr mit supratentorieller Schädigung entweder durch eine zeitversetzte zweite klinische Untersuchung oder durch eine ergänzende apparative Zusatzdiagnostik nachgewiesen werden. 


\section{PRAXIS}

\section{Apnoe-Test}

\section{Zweck}

Nachweis des Ausfalls der Spontanatmung

\section{Ziel}

Provokation der Spontanatmung durch eine gesteigerte $\mathrm{CO}_{2}$-Retention

\section{Voraussetzung}

Ausgangs- $\mathrm{paCO}_{2}$ von $35-45 \mathrm{mmHg}$ in der temperaturkorrigierten Blutgasanalyse (BGA)

\section{Durchführung}

Nachdem der Patient zur Vermeidung einer möglichen Hypoxie der inneren Organe ausreichend präoxygeniert wurde, wird dieser über 4-10 min gezielt, z. B. mit einer Atemfrequenz von 2/min, hypoventiliert. Anschließend wird BGA-kontrolliert ein $\mathrm{paCO}_{2}$-Anstieg bis $>60 \mathrm{mmHg}$ toleriert. Bei Patienten mit chronischer Hyperkapnie gibt es keine definierten $\mathrm{paCO}_{2}$-Grenzwerte. Wichtig ist hierbei, dass die Auswertung der BGAs weiterhin an die Körperkerntemperatur des Patienten angepasst wird.

\section{Auswertung}

Ein zentraler Atemstillstand liegt vor, wenn unter diesem $\mathrm{paCO}_{2}$-Anstieg die Spontanatmung weiterhin ausbleibt.

\section{Stellenwert}

Der Apnoe-Test wird trotz potenzieller Komplikationen wie z. B. kardiovaskulärer Nebenwirkungen und dem Risiko eines Barotraumas weiterhin als fester Bestandteil einer jeden Hirnfunktionsausfallsdiagnostik angesehen [4].

\section{Beobachtungszeitraum}

Vor der Durchführung einer erneuten klinischen Untersuchung zur Bestätigung der Irreversibilität des Hirnfunktionsausfalls muss ein Beobachtungszeitraum eingehalten werden. Der Beobachtungszeitraum, in dem klinische Ausfallsymptome beobachtet werden müssen und der für die Erfüllung der Kriterien eines irreversiblen Hirnfunktionsausfalls Voraussetzung ist, variiert in Abhängigkeit der Art der Hirnschädigung:

- Bei der primären Hirnschädigung gelten für Erwachsene $12 \mathrm{~h}$ Beobachtungszeit.

- Bei der sekundären Hirnschädigung muss die Beobachtungszeit in allen Fällen $72 \mathrm{~h}$ betragen.
Bei Säuglingen und Kindern bis zur Vollendung des 2. Lebensjahres wird ein längerer Beobachtungszeitraum für die Diagnose des irreversiblen Hirnfunktionsausfalls vorausgesetzt:

- Bei Neugeborenen muss die Beobachtungszeit mind. $72 \mathrm{~h}$ betragen.

- Bei Säuglingen und Kleinkindern bis zur Vollendung des 2. Lebensjahres muss die Beobachtungszeit mind. $24 \mathrm{~h}$ betragen.

\section{Merke}

Die Ergänzung der apparativen Diagnostik, die in diesem Fall zwangsläufig erfolgen muss, kann frühestens nach Ablauf des Beobachtungszeitraumes erfolgen.

\section{PRAXIS}

\section{Klinische Zeichen des irreversiblen} Hirnfunktionsausfalls

- Evaluation des Bewusstseinszustandes (Koma)

- Beurteilung der Pupillenweite und Lichtreagibilität ohne Mydriatikum (weit und lichtstarr)

- Ausfall des okulozephalen bzw. des vestibulookulären Reflexes

- Ausfall des Kornealreflexes

- Ausfall der Trigeminusschmerzreaktion

- Ausfall des Pharyngeal- und Trachealreflexes

- Ausschluss einer Spontanatmung im Apnoe-Test

\section{Apparative Diagnostik}

Bei Erwachsenen und Kindern ab dem 2. Lebensjahr mit supratentorieller Schädigung muss die Irreversibilität des Funktionsausfalls entweder durch eine zeitversetzte zweite klinische Untersuchung oder durch eine ergänzende apparative Zusatzdiagnostik nachgewiesen werden. Bei primär infratentoriellen und kombinierten supra- und infratentoriellen Schädigungen kann die Irreversibilität erst bei Vorliegen eines isoelektrischen EEG oder bei nachgewiesenem zerebralem Zirkulationsstillstand bestätigt werden.

Bei Kindern bis zur Vollendung des 2. Lebensjahrs und bei Säuglingen muss in allen Fällen eine ergänzende apparative Diagnostik (entweder ein isoelektrisches EEG oder der Ausfall der frühen akustisch evozierten Potenziale oder der Nachweis des zerebralen Perfusionsstillstands) zur Feststellung der Irreversibilität erfolgen. 


\section{Elektroenzephalogramm}

Im EEG muss unter standardisierten EEG-Ableitungen und Bedingungen über 30 Minuten hinweg eine „hirnelektrische Stille“, auch isoelektrisches EEG genannt, festgestellt werden ( $\mathbf{A b b}$. 1) [5].

\section{Evozierte Potenziale}

Der Hirnfunktionsausfall bei supratentoriellen und sekundären Hirnschädigungen kann mittels des Ausfalls der intrazerebralen Komponente der frühen akustischen Potenziale und der zerebralen und hochzervikalen Komponenten der somatosensibel evozierten Potenziale festgestellt werden [5].

\section{Nachweis eines zerebralen Zirkulationsstillstands}

Der zerebrale Zirkulationsstillstand kann sowohl duplexsonografisch als auch mittels computertomografischer Angiografie (CTA) ermittelt werden [5].

\section{Merke}

Der Perfusionsstillstand tritt ein, sobald der intrakranielle Druck den mittleren arteriellen Druck (MAP) übersteigt und gilt als beweisend für den irreversiblen Funktionsausfall.

Voraussetzung für diese Untersuchungen ist jedoch ein arterieller Mitteldruck $>60 \mathrm{mmHg}$. Unter bestimmten Bedingungen, wie z. B. bei großen offenen Schädel-HirnVerletzungen und bei operativ dekomprimierten Patienten, kann eine regionale Restperfusion, z.B. aufgrund von extrakraniellen Anastomosen, bestehen bleiben. In diesen Fällen muss die Irreversibilität des Hirnfunktionsausfalls entweder durch eine klinische Untersuchung oder eine zeitversetzte erneute apparative Untersuchung nachgewiesen werden.

Die konventionelle Angiografie ist laut den aktuellen Richtlinien, außer es würde sich aus dieser eine mögliche therapeutische Konsequenz ergeben, zur Dokumentation des Zirkulationsstillstandes nicht mehr zugelassen. Dazu muss aber ein mittlerer arterieller Druck von $>80 \mathrm{mmHg}$ bestehen, da ein solcher Zirkulationszustand in den Hirnbasisarterien nur bei ausreichendem systemischen Blutdruck diagnostiziert werden kann.

Nach der Diagnose des irreversiblen Hirnfunktionsausfalls werden die intensivmedizinischen Maßnahmen bei Organspendern zur Organerhaltung fortgeführt. Ist der Patient nicht zur Organspende vorgesehen, müssen die therapeutischen Maßnahmen nun zeitnah eingestellt werden.
}

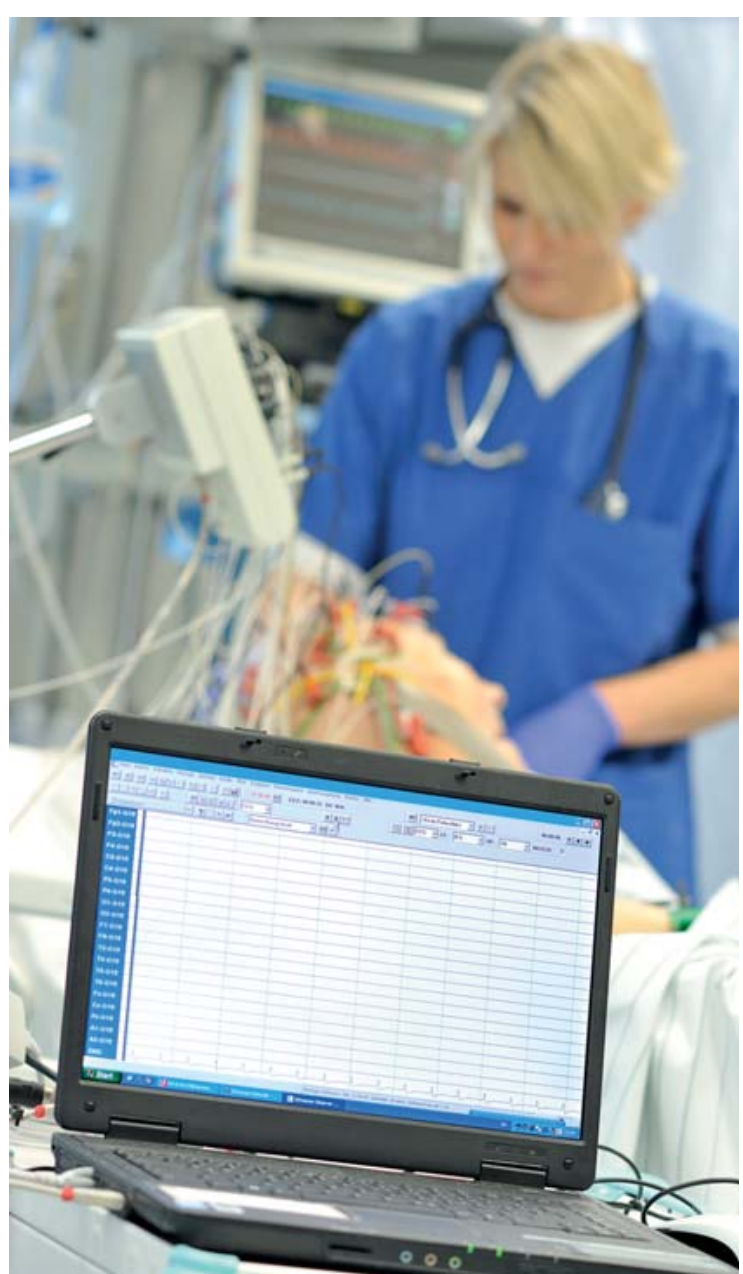

- Abb. 1 Bei primär infratentoriellen und kombinierten supra- und infratentoriellen Schädigungen kann die Irreversibilität des Hirnfunktionsausfalls erst bei Vorliegen eines isoelektrischen EEG oder bei nachgewiesenem zerebralem Zirkulationsstillstand bestätigt werden. Quelle: K.H. Krauskopf.

\section{Todeszeitpunkt und weiteres klinisches Management des potenziellen Organspenders}

In Abhängigkeit des (mutmaßlichen) Patientenwillens muss die intensivmedizinische Therapie bei Patienten, die keiner Organspende zugestimmt hätten, zeitnah beendet werden, da bei fehlender Aussicht auf Heilung oder Leidenslinderung keine Berechtigung für die Fortführung intensivmedizinischer Maßnahmen besteht.

\section{Organerhaltende Behandlung}

Nach der endgültigen Diagnose des Hirntodes beginnt die Phase der organerhaltenden Behandlung für den potenziellen Organspender. Nach Eintritt des irreversiblen Hirnfunktionsausfalls ist häufig eine zentrale Dysregulation der physiologischen Parameter zu verzeichnen. Häu- 


\section{DEFINITION}

\section{Zeitpunkt des Todes}

Als Todeszeitpunkt des Patienten gilt der Zeitpunkt, an dem die Diagnostik des irreversiblen Hirnfunktionsausfalls unter Berücksichtigung der oben aufgeführten Voraussetzungen und Bedingungen abgeschlossen wurde.

\section{FALLBEISPIEL}

Am Folgetag zeigt sich der Thiopental-Spiegel unter der Nachweisgrenze. Die Pupillen bleiben weiterhin weit und lichtstarr. Auch nachdem alle Sedativa über 12 Stunden ausgeschlichen sind, zeigt die Patientin keinerlei Anhalt für Spontanatmung und bleibt komatös.

Bezüglich des zu erwartenden irreversiblen Hirnfunktionsausfalls wird die Deutsche Stiftung Organtransplantation (DSO) informiert und in einem gemeinsam geführten Gespräch mit den Angehörigen der mutmaßliche Patientenwille festgestellt. Anschließend wird mittels Reflexprüfung und dem Apnoe-Test erstmalig der Ausfall der Hirnstammreflexe durch zwei Fachärzte mit langjähriger neurologischer und neurochirurgischer Intensiverfahrung festgestellt.

Nach einem Beobachtungszeitraum von 12 Stunden wird die Prüfung der klinischen Zeichen wiederholt und der Tod der Patientin durch den Nachweis des irreversiblen Hirnfunktionsausfalls festgestellt.

fig kommt es im Rahmen der Hirnschwellung mit Anstieg des intrakraniellen Druckes aufgrund des Cushing-Reflexes (Bradykardie und Hypertonie) zu erheblichen Blutdruckschwankungen und einer hämodynamischen Instabilität, sodass zum Teil sehr hohe Dosen von Katecholaminen benötigt werden. Der steigende Katecholaminbedarf kann wiederum zu einer Minderperfusion der peripheren Organe führen.

\section{PRINZIP}

Nach Feststellung des irreversiblen Hirnfunktionsausfalls sollte nicht mehr der zerebrale Perfusionsdruck, sondern die Erhaltung des peripheren Kreislaufs Ziel der Katecholamintherapie sein. Somit stellt das Katecholamin- und Flüssigkeitsmanagement eine der wesentlichen Herausforderungen bei der Versorgung potenzieller Organspender dar.
- Tab. 1 Zielparameter zur Organerhaltung bei Organspendern.

\begin{tabular}{|c|c|}
\hline Parameter & Zielparameter \\
\hline \multirow{2}{*}{ Blutdruck } & $100-180 \mathrm{mmHg}$ \\
\hline & $\mathrm{MAP}>65 \mathrm{mmHg}$ \\
\hline \multirow[t]{3}{*}{ Respiratio } & $\mathrm{Pa}_{\mathrm{a}} \mathrm{O}_{2}>100 \mathrm{mmHg}$ \\
\hline & $\mathrm{S}_{\mathrm{p}} \mathrm{O}_{2}>95 \%$ \\
\hline & $\mathrm{P}_{\mathrm{a}} \mathrm{CO}_{2} 35-40 \mathrm{mmHg}$ \\
\hline pH-Wert & $7,35-7,45$ \\
\hline Elektrolyte & Natrium $135-145 \mathrm{mmol} / \mathrm{l}$ \\
\hline Temperatur & $>35^{\circ} \mathrm{C}$ \\
\hline Blutzucker & $<200 \mathrm{mg} / \mathrm{dl}$ \\
\hline
\end{tabular}

Respiratorisch entwickeln viele Patienten aufgrund des steigenden Katecholaminbedarfs, des peripheren Gefäßwiderstands und der Volumensubstitution zur Kreislaufstabilisierung eine erhöhte Durchblutung der Pulmonalarterien, was in der Lunge zur Beschädigung des Endothels und letztendlich zum Lungenödem führen kann. Die Beatmung sollte grundsätzlich lungenprotektiv mit geringen Tidalvolumina ähnlich wie bei Patienten mit „Acute Lung Injury“ erfolgen [6].

Mit Ausfall der Funktion der Hypophysen-NebenrindenHormonachse kommt es bei vielen Patienten auch zu Störungen des Stoffwechsels. Potenzielle Organspender neigen zu Hyperglykämien und Elektrolytentgleisungen (insbesondere Hypernatriämien), welche aggressiv zur Organprotektion behandelt werden müssen ( vielen Fällen kommt es durch unzureichende Produktion des antidiuretischen Hormons (ADH) zu einem Diabetes insipidus centralis, welcher durch Gabe von Vasopressin behandelt werden sollte. Zahlreiche Studien haben gezeigt, dass eine organerhaltende Therapie mit hormoneller Substitution die Erhaltung der Organfunktion bei Organspendern begünstigen kann [7]. Aufgrund der Stoffwechselstörung, des Ausfalls des Kältezitterns und einer ungesteuerten peripheren Vasodilatation stellen sich bei Organspendern häufig hypotherme Körperkerntemperaturen ein, welchen frühzeitig durch aktive und passive Wärmung entgegengesteuert werden sollte.

Die Zielparameter zur Organerhaltung bei Organspendern fasst $>$ Tab. 1 zusammen. 


\section{Begleitung der Angehörigen}

Der Umgang und die Kommunikation mit den Angehörigen ist bei potenziellen Organspendern eine ganz besondere Herausforderung für die behandelnden Ärzte. Die Verantwortung, die der behandelnde Arzt gegenüber dem Patienten, seinen Angehörigen und im Rahmen der Organspende trägt, wird zunehmend in der Literatur diskutiert [8].

In vielen Situationen, v. a. bei jüngeren Patienten, wurde eine mögliche Organspende innerhalb der Familie zuvor nicht angesprochen. Hinzu kommt, dass in vielen Fällen die Möglichkeit einer Organspende und die Indikation für eine Diagnose des irreversiblen Hirnfunktionsausfalls bereits kurz nach der Aufnahme auf einer Intensivstation besprochen werden muss. Die Angehörigen, die bereits Schwierigkeiten haben, mit dem Akutereignis umzugehen, sehen sich in solchen Situationen oft überfordert.

Wichtig ist es, die Angehörigen bereits früh in den Entscheidungsprozess mit einzubinden. Sinnvoll ist auch die Vermittlung der schlechten und letztendlich aussichtslosen klinischen Prognose. Der Zeitpunkt, wann eine mögliche Organspende angesprochen werden sollte, hängt sehr von den individuellen Umständen ab, sollte aber erst nach Erfüllung der Voraussetzungen für die Diagnose des irreversiblen Hirnfunktionsausfalls und bei absehbarem Eintritt desselben erfolgen. Im Idealfall sollte das Gespräch vom behandelnden Arzt zusammen mit einem Koordinator der DSO geführt werden.

Ärzte und Pflegende auf der Intensivstation sind die ersten Ansprechpartner für alle Fragen zur Situation des Patienten. Die Koordinatoren der Deutschen Stiftung Organtransplantation (DSO) betreuen den Organspendeprozess und begleiten die Angehörigen in dieser Zeit und auf Wunsch auch darüber hinaus. Die Koordinatoren können von der Klinik hinzugezogen werden, wenn sich die klinischen Zeichen des eintretenden oder zu erwartenden irreversiblen Hirnfunktionsausfalls (IHA) verdichten. Sie beraten sich mit den behandelnden Ärzten über die bisherige Kommunikation mit den Angehörigen und besprechen deren weitere gemeinsame Begleitung.

\section{Gesetzliche Rahmenbedingungen}

Die rechtliche Voraussetzung für eine Organ- und/oder Gewebeentnahme ist die Feststellung des Todes durch Nachweis des irreversiblen Hirnfunktionsausfalls einerseits, die Einwilligung bzw. Zustimmung des Verstorbenen, seiner Angehörigen oder einer diesen gleichgestellten Person andererseits. Im Januar 2020 wurde bezüglich der gesetzlichen Voraussetzungen das „Gesetz zur Stärkung der Entscheidungsbereitschaft bei der Organspende" beschlossen, welches voraussichtlich 2022 in Kraft treten wird. Es handelt sich hierbei weiterhin um
GESETZLICHE RAHMENBEDINGUNGEN

\section{Ermittlung des mutmaßlichen Willens des Verstorbenen durch Angehörige}

Die entscheidungsbefugte Person hat bei ihrer Entscheidung den mutmaßlichen Willen des Verstorbenen zu beachten. Ist der mutmaßliche Wille nicht ermittelbar, erfolgt eine Entscheidung nach den Wertvorstellungen der entscheidungsbefugten Person.

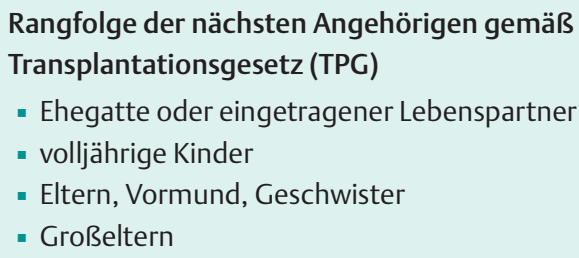

Ist kein Angehöriger oder keine nahestehende Person des Verstorbenen eruierbar, findet im Sinne der Entscheidungslösung (Zustimmungslösung) keine Organspende statt.

eine Zustimmungslösung. Dabei wurde auch eine umfassendere und regelmäßige Aufklärung der Bürger über die Möglichkeit der Organspende beschlossen.

Um den Willen des Verstorbenen bezüglich der Organspende zu eruieren, muss zuerst geprüft werden, ob eine Entscheidung des Verstorbenen zur Organspende schriftlich dokumentiert vorliegt oder mündlich bekannt ist. Dabei geht es nicht nur um die grundsätzliche Entscheidung, sondern auch um den Umfang einer möglichen Spende bzw. Einschränkungen, die getroffen wurden. Die Entscheidung des Verstorbenen ist grundsätzlich bindend. Für den Fall, dass die Angehörigen aber berichten, dass der Verstorbene seine Einstellung aktuell geändert hatte, ist das entsprechend zu berücksichtigen.

Wenn keine Entscheidung bekannt ist, liegt es bei den Angehörigen, nach dem mutmaßlichen Willen des Verstorbenen (s. Infobox) eine Entscheidung treffen. Dabei hat das Transplantationsgesetz (TPG) eine Rangfolge der nächsten Angehörigen bzw. gleichgestellten Personen festgelegt. Voraussetzung ist dabei immer, dass es in den vorhergehenden zwei Jahren einen persönlichen Kontakt gab.

Wurde die Entscheidung vom Verstorbenen zu Lebzeiten auf eine andere Person übertragen, so tritt diese an die Stelle des nächsten Angehörigen.

In Deutschland basiert die Entscheidung für oder gegen eine Organspende am häufigsten auf der Grundlage des 


\section{Dokumentation von Ablauf, Inhalt und Ergebnis der Beteiligung der Angehörigen oder gleichgestellter Personen}

$\S 11$ Abs. 1a Nr. 3 TPG zur Überprüfung der Einzelheiten der Einwilligung des Spenders nach §3 TPG oder der Zustimmung anderer Personen nach §4 TPG

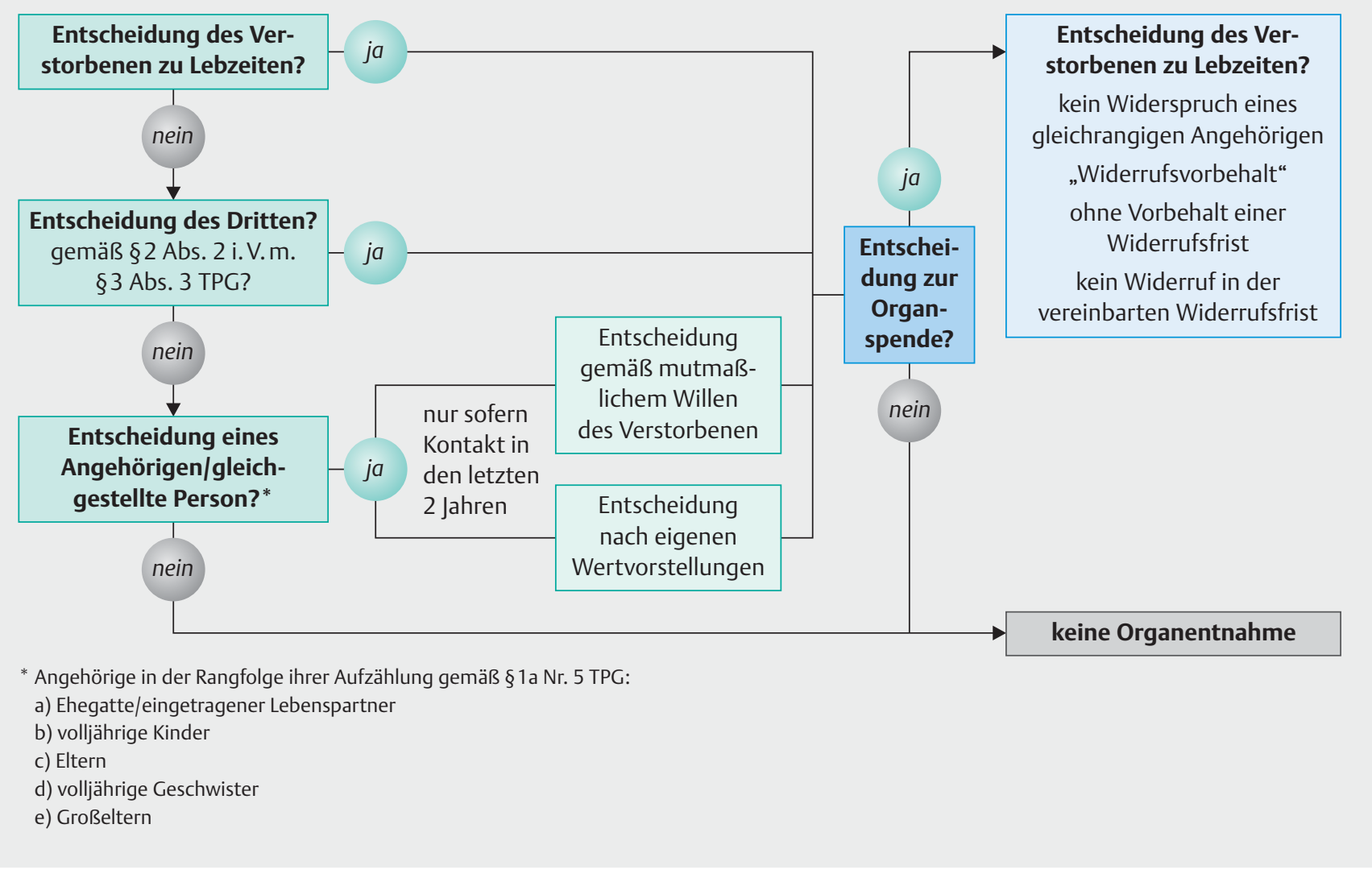

- Abb. 2 Überprüfung der Einzelheiten der Einwilligung des Spenders nach § 3 TPG (Transplantationsgesetz) oder der Zustimmung anderer Personen nach § 4 TPG (§ 11 Abs. 1a Nr. 3 TPG). Quelle: Deutsche Stiftung Organtransplantation (DSO). Quelle: Deutsche Stiftung Organtransplantation.

mutmaßlichen Willens des Verstorbenen, der von den Angehörigen eingeschätzt wird ( $\triangleright$ Abb. 2).

In den Fällen, in denen keine Angehörigen auszumachen sind, darf in Deutschland, wo die Entscheidungslösung gesetzlich gilt, die eine Sonderform der Zustimmungslösung ist, keine Organspende erfolgen.

Ein weiterer wichtiger Aspekt ist, dass die Aufgaben und Verpflichtungen eines Betreuers mit dem Tod der zu betreuenden Person enden und dieser somit nicht in den Entscheidungsprozess involviert werden kann. Eine Ausnahme besteht, wenn gleichzeitig eine besondere persönliche Verbundenheit zwischen Betreuer und betreuter Person bestand.

Aktuell gilt in Deutschland die Entscheidungslösung. Diese wird allerdings ab 2022 vom „Gesetz zur Stärkung der Entscheidungsbereitschaft bei der Organspende“ abge- löst. Dieses sieht weiterhin eine klare Zustimmung von Seiten des Verstorbenen bzw. dessen Angehörigen vor, aber auch eine umfassende und regelmäßige Aufklärung der Bürger z. B. durch die Hausärzte bzw. in öffentlichen Ämtern. Außerdem wird das Thema Organspende in den Lehrstoff des Medizinstudiums integriert sowie in die obligaten Erste-Hilfe-Kurse der Autoführerschein-Ausbildung aufgenommen.

\section{Gespräch mit den Angehörigen von möglichen Organspendern}

Ziel des Angehörigengesprächs ist es, zum einen den Angehörigen alle gewünschten Informationen zum Thema Organspende zu vermitteln und zum anderen die Familie bei der Entscheidungsfindung ergebnisoffen zu begleiten, um so eine stabile Entscheidung im Sinne des Verstorbenen zu finden, mit der auch die Hinterbliebenen 
unbelastet weiterleben können. Im Idealfall sollte das Gespräch vom behandelnden Arzt zusammen mit einem Koordinator der DSO geführt werden. Die frühzeitige Hinzunahme eines DSO-Koordinators hat den Vorteil, dass dieser den Familienmitgliedern ausführlich und ohne Zeitdruck zur Verfügung steht und umfassende Informationen zu den Abläufen einer Organspende und Transplantation aus den unterschiedlichen Perspektiven vermittelt.

\section{Merke}

Wichtig ist dabei, dass den Angehörigen genügend Zeit und Raum gegeben wird, um über die Situation und die Frage nach einer Organspende nachzudenken bzw. diese Frage miteinander zu besprechen. Sie müssen die Möglichkeit haben, über ihre Trauer, ihre Ängste oder Sorgen sprechen zu können. Die Entscheidung sollte keinesfalls hastig oder übereilt getroffen werden.

Ablauf, Inhalt und Ergebnis des Gesprächs sowie beteiligte Personen sind vom gesprächsführenden Arzt zu dokumentieren. Hierfür stellt die DSO eine Leitlinie als Formular zur Verfügung [9].

Falls eine Einwilligung zur Organentnahme gegeben wird, sollte die Familie genaue Informationen erhalten, wie das weitere Prozedere aussieht. Dies reicht von dem Umfang der für die Spendercharakteristika notwendigen Untersuchungen, dabei möglicherweise erhobenen unerwarteten Befunden und ihren möglichen Konsequenzen, der Mitteilung des geplanten OP-Zeitpunktes, bis zu weiteren logistischen Informationen. Das Ausmaß der Informationen hängt dabei immer vom Wunsch und den Bedürfnissen der Angehörigen ab.

Im weiteren Verlauf sollte gegenüber den Angehörigen weiter Gesprächsbereitschaft signalisiert werden, da v.a. bei protrahierten Verläufen, bei denen die Feststellung des Hirnfunktionsausfalls mehrere Tage dauern kann, Fragen und Zweifel bei den Angehörigen entstehen können. Letztendlich sollten die Angehörigen darauf hingewiesen werden, dass der Prozess der Organspende zu jeder Zeit beendet und die Entscheidung widerrufen werden kann.

Besonders alarmierend für Angehörige sind verbleibende spinale Reflexe des Patienten, welche den Anschein einer Restfunktion des Gehirns und v. a. einer bewussten Reaktion des Patienten erwecken können [10]. Spinale Reflexe können sich in verschiedenen Formen von Muskelzuckungen bis hin zu ungerichteten Bewegungen und Erektionen manifestieren und z. B. durch taktile Reize provoziert werden, sodass diese durch den Laien schnell als Lebenszeichen fehlinterpretiert werden. Daher sollten Angehörige frühzeitig über das Auftreten von spinalen Reflexen aufgeklärt werden. Diese Reflexe werden ausschließlich auf Ebene des Rückenmarks verschaltet und stehen somit nicht im Zusammenhang mit einer kortikalen oder zerebralen Restfunktion.

Ein besonderes Augenmerk des DSO-Koordinators liegt auf dem Hinweis und dem Angebot der Möglichkeit, sich von dem Verstorbenen nach der Organentnahme zu verabschieden. Das Transplantationsgesetz schreibt vor, dass den Angehörigen diese Möglichkeit obligat genannt werden muss. Es ist für viele Angehörige von großer Bedeutung, sich selbst noch einmal von dem würdevollen Zustand ihres Verstorbenen nach der Organentnahme zu überzeugen. Bei DSO-Umfragen von Angehörigen von Organspendern war die Angst vor Entstellung des Verstorbenen durch die Organentnahme eine der häufig genannten Bedenken. Aber auch medizinisches Personal in den Kliniken spricht diesen Aspekt häufig an.

\section{RESÜMEE}

Der DSO-Koordinator informiert die Angehörigen des Organspenders über den primären Verlauf der Operation und unterstützt das Klinikpersonal bei der postoperativen Abschiednahme.

\section{Angebote zur weiteren Betreuung}

In der Novellierung des Transplantationsgesetzes vom April 2019 wurde aufgenommen, dass im Rahmen eines akuten Organspendeprozesses die Angehörigen vom Koordinator der DSO Informationen zu weiteren Angeboten zur Unterstützung über den Organspendeprozess hinaus erhalten sollen. Dazu gehört die Kontaktmöglichkeit bei nachträglich auftretenden Fragen. Auf Wunsch erhält die Familie ca. 6 Wochen nach der Spende einen Brief mit anonymen Informationen über die Empfänger und den Verlauf der Transplantation. Darüber hinaus bieten die Regionen der DSO Angehörigentreffen an. Diese Treffen finden 2- bis 3-mal im Jahr pro Region statt und werden von Psychologen und DSO-Mitarbeitern begleitet. Bei diesen Treffen tauschen sich die Angehörigen von Organspendern mit anderen Angehörigen und vor allem auch Transplantierten aus.

\section{Entscheidungsbegleitung für Angehörige (EfA)}

Die DSO bietet Fortbildungen an, die die Mitarbeiter in Kliniken/auf Intensivstationen, die Angehörige von Organspendern begleiten, auf die Gespräche mit Angehörigen von möglichen Organspendern vorbereiten. Dazu wurde unter fachkundiger psychologischer Beratung ein Fortbildungsangebot entwickelt. Speziell geschulte DSOKoordinatoren organisieren Seminare und stehen für klinikinterne Veranstaltungen zur Verfügung.

Die Teilnehmer erhalten Grundlagen zur Führung eines Angehörigengesprächs. Die Seminarinhalte sind ein Mix 
aus fundiertem Hintergrundwissen und Erfahrungsberichten der Koordinatoren. Zusätzlich werden anschauliche Beispiele anhand von Lehrfilmen gezeigt oder mit Schauspielern Gespräche nachgestellt und trainiert.

Merke

Die Ansprechpartner bei der DSO informieren über Art und Umfang der Schulungen „Entscheidungsbegleitung für Angehörige (EfA)“ (www.dso.de).

\section{Spendercharakterisierung}

Liegt eine Einwilligung zur Organspende vor, werden bei dem Verstorbenen intensivmedizinische Maßnahmen bis zum Zeitpunkt der Entnahme fortgeführt, damit die Funktion der Organe für die späteren Empfänger erhalten bleibt (s. o.: Abschn. „Todeszeitpunkt und weiteres klinisches Management des potenziellen Organspenders“).

Jede Transplantation birgt neben dem grundsätzlichen immunologischen Risiko einer Abstoßung auch Risiken im Hinblick auf die potenzielle Übertragung von malignen Erkrankungen, Infektionskrankheiten, genetisch bedingten Erkrankungen oder toxischen Schädigungen. Um die Risiken für die Übertragung von Krankheiten möglichst auszuschließen, müssen bestimmte bildgebende und laborchemische Untersuchungen obligat durchgeführt werden, die im Rahmen der Richtlinie zur Spendercharakterisierung der Bundesärztekammer festgelegt und regelmäßig aktualisiert werden. Abhängig von der individuellen Situation des Organspenders können darüber hinausgehende Untersuchungen notwendig sein.

Die DSO-Koordinatoren veranlassen gemeinsam mit den betreuenden Intensivärzten alle notwendigen medizinischen Untersuchungen. Ziel dieser sorgfältigen Untersuchung und Charakterisierung des Spenders ist es, mögliche Erkrankungen und Infektionen des Spenders zu erkennen, die die Empfänger gefährden könnten. In Laboruntersuchungen werden außerdem die Blutgruppe und Gewebemerkmale bestimmt. Beides sind wichtige Daten für die Vermittlung der Organe.

\section{Zeitpunkt der Untersuchung}

Die Erhebung der Anamnese, die körperliche Untersuchung sowie eine Reihe weiterer Laboruntersuchungen und apparativer Diagnostiken sind die Basis der Organund Spendercharakterisierung.

Die Organ- und Spendercharakterisierung ist ein stufenweiser Prozess. Befunde, die vor der Feststellung des irreversiblen Hirnfunktionsausfalls im Rahmen der Behandlung des Patienten erhoben wurden, können zur Organund Spendercharakterisierung hinzugezogen werden. Generell sind bei der Beurteilung der jeweiligen Organ- funktion die zum Zeitpunkt der Befunderhebung vorliegenden Umstände sowie im Rahmen der Intensivtherapie seitdem durchgeführten Maßnahmen zu berücksichtigen. Gegebenenfalls kann eine Wiederholung der Untersuchung angezeigt sein.

Die DSO hält im „Leitfaden für die Organspende“ [9] und in den Verfahrensanweisungen gemäß $§ 11$ des Transplantationsgesetzes [11] Informationen und notwendige Formulare für den Ablauf der Spendercharakterisierung bereit.

Die erhobenen Befunde dienen als medizinische Entscheidungsgrundlage für:

- den Umfang der Organspende

- den Empfängerschutz

- die Allokation

- die Akzeptanz des vermittelten Organs

\section{GESETZLICHE RAHMENBEDINGUNGEN}

\section{Vorbereitung einer Organentnahme}

Maßnahmen, die ausschließlich der Vorbereitung einer Organentnahme dienen, dürfen erst und nur dann vorgenommen werden, wenn der irreversible Hirnfunktionsausfall festgestellt worden ist und eine Zustimmung zur Organentnahme vorliegt.

\section{Meldung an die Vermittlungsstelle Eurotransplant}

Zusammen mit weiteren Angaben zum Organspender übermitteln die DSO-Koordinatoren die Untersuchungsergebnisse an die Vermittlungsstelle Eurotransplant. Dort gleicht ein Computerprogramm die medizinischen Daten des Spenders mit denen der Patienten auf den Wartelisten ab und ermittelt die passenden Empfänger. Bei der Vergabe der Spenderorgane spielen Aspekte wie Dringlichkeit, Gewebeübereinstimmung und Erfolgsaussicht eine wichtige Rolle. Die Richtlinien für die Organvermittlung erstellt die Bundesärztekammer [12].

\section{Entnahme der Organe}

Im Rahmen der postmortalen Organspende können Nieren, Herz, Leber, Lunge, Pankreas und Dünndarm für eine Transplantation gespendet werden. In der Regel entnehmen die Chirurgen, die auch die spätere Transplantation durchführen, Herz und Lunge. Nieren, Pankreas und Leber entnehmen in den meisten Fällen regionale Entnahmeteams. 
Merke

Die DSO organisiert die Entnahmeteams für die jeweiligen Organe.

Bei der Organentnahme ist der respektvolle Umgang mit dem Spender oberstes Gebot. Die Organspende erfolgt unter den gleichen Bedingungen wie jede andere Operation. Die Ärzte verschließen die Operationswunde sorgfältig und übergeben den Spender in würdigem Zustand. Die Angehörigen können sich nach der Organentnahme in gewünschter Weise von dem Verstorbenen verabschieden.

\section{Angebote der DSO \\ für die Krankenhäuser}

Als Koordinierungsstelle bietet die DSO den Krankenhäusern, insbesondere den Transplantationsbeauftragten, vielfältige Unterstützungsmöglichkeiten an. Dazu gehören Schulungen sowie individuelle Bedarfsanalysen für einzelne Krankenhäuser. Zudem hat die DSO spezifische Unterstützungsangebote entwickelt, etwa den Leitfaden für die Organspende sowie die Verfahrensanweisungen, die Handlungssicherheit im Organspendeprozess vermitteln. Ergänzt wird das Angebot durch ein umfangreiches und von den Landesärztekammern anerkanntes E-Learning-Fortbildungsprogramm, zu dem auch ein „virtueller Spender" gehört, der die Anwendung des erworbenen Wissens in einer Übungssituation ermöglicht.

\section{PRAXIS}

Zentrale Unterstützungs- und Schulungsangebote der DSO für Krankenhausmitarbeiter

- direkte Unterstützung im Organspendeprozess

- Beratungs- und Fortbildungsangebot für Klinikmitarbeiter

- Verfahrensanweisungen

- Leitfaden Organspende

- TransplantCheck für Excel

- DSO.isys ${ }^{+}$

- E-Learning (Grundlagen der Organspende, virtueller Spender)

\section{KERNAUSSAGEN}

- Der Hirntod ist definiert als nicht behebbarer Ausfall der Gesamtfunktion des Großhirns, des Kleinhirns und des Hirnstamms (irreversibler Hirnfunktionsausfall).

- Die Hirntodfeststellung erfolgt auf Basis der aktuellsten Fortschreibung der Richtlinie der Bundesärztekammer anhand eines Drei-Stufen-Schemas und besteht aus klinischen und apparativen Untersuchungen.

- Nach der endgültigen Diagnose des Hirntodes beginnt die Phase der organerhaltenden Behandlung für den potenziellen Organspender. Bei Patienten, die selbst oder deren Angehörige keiner Organspende zugestimmt haben, muss die intensivmedizinische Therapie zeitnah beendet werden.

- Die gesetzlichen Rahmenbedingungen zur Feststellung des Hirntodes und zur Organentnahme potenzieller Organspender gibt das Transplantationsgesetz vor.

- Die Deutsche Stiftung Organtransplantation (DSO) ist in Deutschland zuständig für die Koordinierung von Organspenden.

- Die DSO unterstützt die Krankenhäuser in vielfältiger Weise während des Organspendeprozesses aber auch bei Fortbildungen zum Thema Organspende des medizinischen Personals. Der Hauptansprechpartner der DSO ist hierbei der Transplantationsbeauftragte in den Krankenhäusern.

- Der Betreuung der Angehörigen eines potenziellen Organspenders kommt große Bedeutung zu.

Interessenkonflikt

Erklärung zu finanziellen Interessen

Forschungsförderung erhalten: nein; Honorar/geldwerten Vorteil für Referententätigkeit erhalten: nein; Bezahlter Berater/interner Schulungsreferent/Gehaltsempfänger: nein; Patent/Geschäftsanteile/Aktien (Autor/Partner, Ehepartner, Kinder) an im Bereich der Medizin aktiven Firma: nein; Patent/ Geschäftsanteile/Aktien (Autor/Partner, Ehepartner, Kinder) an zu Sponsoren dieser Fortbildung bzw. durch die Fortbildung in ihren Geschäftsinteressen berührten Firma: nein.

Erklärung zu nichtfinanziellen Interessen

Die Autorinnen/Autoren geben an, dass kein Interessenkonflikt besteht. 


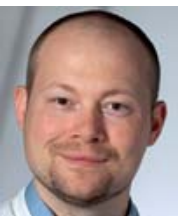

\section{Matthias N. Ungerer}

Dr. med. Seit April 2014 Assistenzarzt in der neurologischen Abteilung der Universitätsklinik Heidelberg. Klinischer Schwerpunkt: neurologische Akut- und Intensivmedizin.

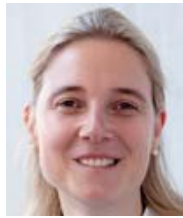

\section{Silvia Schönenberger}

PD Dr. med. Oberärztin der Neurologischen Intensivstation am Universitätsklinikum Heidelberg, Klinik für Neurologie. Zusatzbezeichnung und volle Weiterbildungsberechtigung für die Zusatzweiterbildung Intensivmedizin.

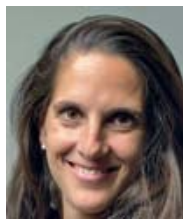

\section{Ana Paula Barreiros}

PD Dr. med. Seit 2015 Geschäftsführende Ärztin der Deutschen Stiftung Organtransplantation (DSO) in der Region Mitte. 2010 Habilitation im Bereich Innere Medizin. Vor Tätigkeit für die DSO Oberärztin u. a. in der Universitätsmedizin Mainz und am Universitätsklinikum Regensburg und dort u. a. für die Versorgung von Lebertransplantierten zuständig. Seit 2007 Dozentin des Masterstudiengangs „Medizinethik“ der Universitätsmedizin Mainz.

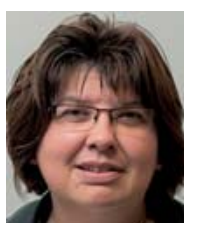

\section{Anne-Bärbel Blaes-Eise}

Fachkrankenschwester für Nephrologie. Seit 1994 für die Deutsche Stiftung Organtransplantation tätig, seit 2000 als Koordinatorin in der Organspende. Schwerpunkt: Begleitung von Spenderfamilien in der Akutsituation, besonders jedoch in der Folgezeit. Leiterin zahlreicher Workshops für Angehörige und medizinisches Personal.

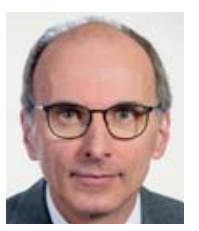

\section{Axel Rahmel}

Dr. med. Seit 2014 Medizinischer Vorstand der Deutschen Stiftung Organtransplantation. 1997-2005 Oberarzt in der Klinik für Herzchirurgie und der Klinik für Kardiologie am Herzzentrum der Universität Leipzig; 20052014 Medizinischer Direktor der Eurotransplant International Foundation, Leiden, Niederlande. Seit 2003 Mitglied der „Ständigen Kommission Organtransplantation“ der Bundesärztekammer.

\section{Korrespondenzadresse}

\section{Dr. med. Matthias Ungere}

Neurologische Klinik, Universität Heidelberg Im Neuenheimer Feld 400, 69120 Heidelberg matthias.ungerer@med.uni-heidelberg.de

\section{Wissenschaftlich verantwortlich gemäß Zertifizierungsbestimmungen}

Wissenschaftlich verantwortlich gemäß Zertifizierungsbestimmungen für diesen Beitrag ist Dr. med. Silvia Schönenberger, Heidelberg.

\section{Zitierweise für diesen Artikel}

Anasthesiol Intensivmed Notfallmed Schmerzther 2020; 55; 453-466. doi:10.1055/a-1167-1420

Dieser Beitrag ist eine aktualisierte Version des Artikels: Ungerer MN, Schönenberger S, Barreiros AP et al. Hirntod und Umgang mit (potenziellen) Organspendern und Angehörigen. Intensivmedizin up2date 2018; 14: 435-447.

Literatur

[1] Richter-Kuhlmann E. Neues Transplantationsgesetz: Der richtige Ansatz. Dtsch Arztebl 2019; 116: A-123/B-107/C-107

[2] Klinkhammer G, Richter-Kuhlmann E. Richtlinie zur Feststellung des Hirnfunktionsausfalls: Neuer Titel, präzisierte Regeln. Dtsch Arztebl 2015; 112: A1230/B-1028/C-1000

[3] Brandt SA, Walter U, Reimers CD et al. Erhebung der klinischen Befunde des irreversiblen Hirnfunktionsausfalls (Hirntod): Voraussetzungen, Durchführung und pathophysiologische Grundlagen. Klin Neurophysiol 2016; 47: 5-15

[4] Machado C, Perez J, Scherle C et al. Brain death diagnosis and apnea test safety. Ann Indian Acad Neurol 2009; 12: 197-200. doi:10.4103/0972-2327.56326

[5] Walter U, Brandt SA, Ferbert A et al. Empfehlungen der Deutschen Gesellschaft für Klinische Neurophysiologie und Funktionelle Bildgebung zur Diagnostik des irreversiblen Hirnfunktionsausfalls. Klin Neurophysiol 2016; 47: 16-20

[6] Kumar L. Brain death and care of the organ donor. J Anaesthesiol Clin Pharmacol 2016; 32: 146-152. doi:10. 4103/0970-9185.168266

[7] Meyfroidt G. Gunst J, Martin-Loeches I et al. Management of the brain-dead donor in the ICU: general and specific therapy to improve transplantable organ quality. Intensive Care Med 2019; 45: 343-353

[8] Shemie SD, Simpson C, Blackmer J et al. Ethics guide recommendations for organ-donation-focused physicians: endorsed by the Canadian Medical Association. Transplantation 2017; 101: (5S Suppl 1): S41-S47. doi:10.1097/ TP.0000000000001694

[9] Deutsche Stiftung Organtransplantation - DSO. Leitfaden für die Organspende, Deutsche Stiftung Organtransplantation, 2017. Im Internet: www.dso.de; Stand: 06.10.2018

[10] Linos K, Fraser J, Freeman WD et al. Care of the brain-dead organ donor. Curr Anaesth Crit Care 2007; 18: 284-294

[11] Deutsche Stiftung Organtransplantation - DSO. Verfahrensanweisungen der DSO gemäß § 11 des Transplantationsgesetzes. Im Internet: www.dso.de; Stand: 06.10.2018

[12] Bundesärztekammer. Richtlinien für die Wartelistenführung und die Organvermittlung gem. $\S 16$ Abs. 1 S. 1 Nrn. 2 u. 5TPG. Im Internet: https://www.bundesaerztekammer.de/ richtlinien/richtlinien/transplantationsmedizin/richtlinien-fuer-die-wartelistenfuehrung-und-die-organvermittlung/; Stand: 06.10 .2018

Bibliografie

DOI https://doi.org/10.1055/a-1167-1420

Anästhesiol Intensivmed Notfallmed Schmerzther 2020; 55: 453-466 @ Georg Thieme Verlag KG Stuttgart · New York ISSN 0939-2661 


\section{Punkte sammeln auf CME.thieme.de}

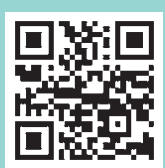

Diese Fortbildungseinheit ist in der Regel 12 Monate online für die Teilnahme verfügbar.

Den genauen Einsendeschluss finden Sie unter https://cme.thieme.de/.

Sollten Sie Fragen zur Online-Teilnahme haben, finden Sie unter https://cme.thieme.de/hilfe

eine ausführliche Anleitung. Wir wünschen viel Erfolg beim Beantworten

der Fragen!

Unter eref.thieme.de/ZZX91YN oder über den QR-Code kommen Sie

direkt zur Startseite des Wissenstests.

VNR 2760512020158722989

\section{Frage 1}

Welche der folgenden Aussagen zur Qualifikation der Ärzte für die Diagnose des irreversiblen Hirnfunktionsausfalls bei einem 17-jährigen Patienten trifft nicht zu?

A Beide Ärzte müssen eine Facharztweiterbildung abgeschlossen haben.

B Mindestens einer der Ärzte muss eine Facharztweiterbildung im Bereich der Pädiatrie abgeschlossen haben.

C Einer der Ärzte muss eine abgeschlossene Facharztausbildung als Neurologe oder Neurochirurg haben.

D Beide Ärzte müssen über eine mehrjährige Erfahrung mit Patienten mit schweren Hirnschädigungen verfügen.

E Einer der Ärzte kann eine Facharztausbildung im Bereich der Pädiatrie mit langjähriger Intensiverfahrung haben.

\section{Frage 2}

Unter welchen Umständen ist eine apparative Zusatzdiagnostik zur Diagnose des irreversiblen Hirnfunktionsausfalls zwangsläu-

fig notwendig?

A grundsätzlich bei allen minderjährigen Patienten

B bei allen Patienten, die mit langwirksamen Sedativa (z. B. Trapanal) behandelt wurden

C bei Patienten mit primär infratentoriellen Hirnschäden

D im Falle des V.a. einen sekundären Hirnschaden (z. B. Hypoxie)

E bei Kleinkindern nach dem 2. Lebensjahr

\section{Frage 3}

Welche der folgenden Aussagen zum Umgang mit den Angehörigen im Rahmen einer möglichen Organspende ist falsch?

A Das 2. Gesetz zur Änderung des Transplantationsgesetzes von 2019 schafft eine klare rechtliche Grundlage für den Austausch von anonymisierten Schreiben zwischen Organempfängern und den nächsten Angehörigen der Organspender.

B Entwickelt ein Patient mit hoher Wahrscheinlichkeit einen irreversiblen Hirnfunktionsausfall und kommt somit ggf. für eine Organspende in Frage, sollte ein ausführliches Gespräch mit den Angehörigen über den mutmaßlichen Patientenwillen vor Einleitung der weiteren Diagnostik erfolgen.
C Im Rahmen eines akuten Organspendeprozesses sollen die Angehörigen vom Koordinator der DSO Informationen zu weiteren Angeboten zur Unterstützung über den Organspendeprozess hinaus erhalten.

D Das Gespräch zur Aufklärung über die Organspende mit den Angehörigen hat der DSO-Koordinator, nicht der betreuende Klinikarzt zu führen.

E Es ist wichtig, Angehörigen genügend Zeit und Raum zu geben, um über die Situation und die Frage nach einer Organspende nachzudenken.

\section{Frage 4}

Die Novellierung des 2. Gesetzes zur Änderung des Transplantationsgesetzes von 2019 umfasst zahlreiche Neuerungen. Welche gehört nicht dazu?

A Bundesweit bzw. flächendeckend wird ein neurologischer/ neurochirurgischer konsiliarärztlicher Rufbereitschaftsdienst eingerichtet.

B Ein neues klinikinternes Qualitätssicherungssystem schafft die Grundlage für ein flächendeckendes Berichtssystem bei der Spendererkennung und Spendermeldung. Dabei sollen die Gründe für eine nicht erfolgte Feststellung des irreversiblen Hirnfunktionsausfalls oder eine nicht erfolgte Meldung an die Koordinierungsstelle (DSO) intern erfasst und bewertet werden.

C Entnahmekrankenhäuser werden künftig für den gesamten Prozessablauf einer Organspende besser vergütet.

D Es erfolgt ein Wechsel von der Zustimmungs- zur Widerspruchslösung.

E Es gibt verbindliche Vorgaben für die Freistellung der Transplantationsbeauftragten.

- Weitere Fragen auf der folgenden Seite... 


\section{Punkte sammeln auf CME.thieme.de}

Fortsetzung $\ldots$

\section{Frage 5}

Welche dieser Zielparameter ist nicht Teil der organerhaltenden Therapie bei Organspendern?
A systolischer Blutdruck $>100 \mathrm{mmHg}$
B $\mathrm{P}_{\mathrm{a}} \mathrm{O}_{2}>100 \mathrm{mmHg}$
C zerebraler Perfusionsdruck $>60 \mathrm{mmHg}$
D Körperkerntemperatur $>35^{\circ} \mathrm{C}$
E mittlerer arterieller Druck $>65 \mathrm{mmHg}$

\section{Frage 6}

Welche Aussage zum Apnoe-Test ist falsch?

A Die BGAs müssen an die Körperkerntemperatur angepasst werden.

B Das Erreichen eines $\mathrm{p}_{\mathrm{a}} \mathrm{CO}_{2}$ von $55 \mathrm{mmHg}$ ist beim Apnoe-Test ausreichend.

C Die Dauer der Hypoventilation sollte 4-10 min betragen.

D Der Patient erhält während des Apnoe-Tests weiterhin Sauerstoff.

E Ein zentraler Atemstillstand liegt vor, wenn eine Spontanatmung des Patienten ausbleibt.

\section{Frage 7}

Der mutmaßliche Patientenwille hinsichtlich einer Organspende kann gemeinsam mit Angehörigen ermittelt werden, wenn der Patient selbst sich dazu nicht mehr äußern kann. Der Gesetzgeber hat dazu welche mögliche Rangfolge festgelegt?

A Bruder - Tochter - Eltern - Großmutter

B Ehegatte - Eltern - Großeltern - leibliches Kind

C eingetragener Lebenspartner - volljähriges Kind - Geschwister

D Eltern - volljähriges Kind - Geschwister

E Vater-Großmutter-Adoptivtochter - eingetragene Lebenspartnerin

\section{Frage 8}

Welche der folgenden Aussagen ist falsch?

A Ist kein Angehöriger oder keine nahestehende Person des Verstorbenen zu ermitteln, findet keine Organspende statt.

B Hatte der Patient noch zu Lebzeiten die Entscheidung zur Organspende auf eine andere Person übertragen, so tritt diese Person an die Stelle des nächsten Angehörigen.

C Angehörigen können den Prozess der Organspende zu jeder Zeit beenden und die Entscheidung widerrufen.

D Ein zu Lebzeiten bestellter gesetzlicher Betreuer muss in den Entscheidungsprozess für oder gegen eine Organspende involviert werden.

E Die Entscheidung, Organspender zu werden, erfolgt auf freiwilliger Basis.

\section{Frage 9}

Nach der ersten klinischen Untersuchung muss vor der erneuten klinischen Untersuchung zur Bestätigung der Irreversibilität des Hirnfunktionsausfalls welcher Beobachtungszeitraum eingehalten werden?

A keiner, wenn auf eine apparative Diagnostik ausgewichen werden kann

B bis zu dem Zeitpunkt, an dem die Angehörigen die Einwilligung unterschrieben haben

C max. 12 Stunden, um die Organschädigung möglichst gering zu halten

D max. 24 Stunden

E mind. 72 Stunden bei Neugeborenen und mind. 24 Stunden bei Kindern bis zum Alter von 2 Jahren

\section{Frage 10}

Welcher Reflexausfall gehört nicht zu den klinischen Zeichen des irreversiblen Hirnschadens?
A vestibulookulärer Reflex
B Rückziehreflex des Beins
C Kornealreflex
D Trigeminusschmerzreaktion
E Trachealreflex 